\title{
Lactobacillus mucosae and Bifidobacterium longum Synergistically Alleviate Immobilization Stress-Induced Anxiety/Depression in Mice by Suppressing Gut Dysbiosis ${ }^{\text {[ }}$
}

\author{
Sang-Kap Han and Dong-Hyun Kim*
}

Neurobiota Research Center, Department of Life and Nanopharmaceutical Sciences, College of Pharmacy, Kyung Hee University, Seoul 02447, Republic of Korea

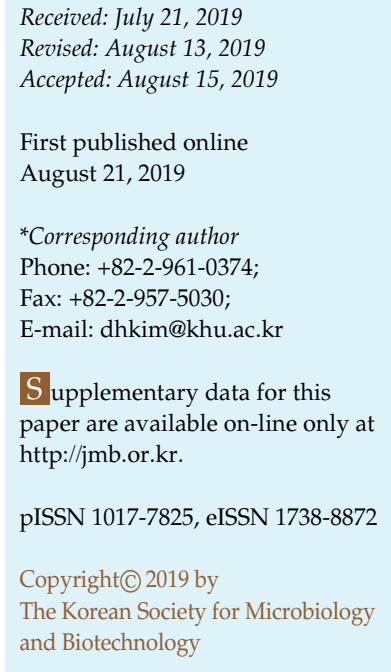

We isolated Lactobacillus mucosae NK41 and Bifidobacterium longum NK46 from human feces, which induced BDNF expression in corticosterone-stimulated SH-SY5Y cells, and examined their anti-depressive effects in mice. NK41, NK46, and their (1:1) mixture significantly mitigated immobilization stress (IS)-induced anxiety-like/depressive behaviors, hippocampal NF- $\kappa \mathrm{B}$ activation, BDNF expression, $\mathrm{Iba}^{+}$cell population, and blood corticosterone, TNF- $\alpha$, IL6 , and lipopolysaccharide levels. Furthermore, they inhibited colitis marker NF- $\mathrm{kB}$ activation, and TNF- $\alpha$ expression in mice with IS-induced anxiety/depression. They additionally suppressed gut Proteobacteria and Bacteroidetes populations and bacterial lipopolysaccharide production. These findings suggest that NK41 and NK46 may alleviate anxiety/depression and colitis by suppressing gut dysbiosis.

Keywords: Bifidobacterium longum, Lactobacillus mucosae, depression, anxiety, gut microbiota
Anxiety disorders are the most commonly reported mental disorders [1]. If not treated, patients with chronic anxiety disorders progress toward depression [2]. In mice, anxiety/depression can be induced by stressors such as immobilization, forced swimming, and antibiotics [3]. Exposure to immobilization stress (IS) stimulates the secretion of adrenal hormones such as glucocorticoids and proinflammatory cytokines such as interleukin (IL)-6 and gut dysbiosis via the activation of the hypothalamopituitary-adrenal (HPA) axis, while brain-derived neurotrophic factor (BDNF) expression is suppressed [4, 5]. However, gut dysbiosis causes gastrointestinal inflammation and increases anxiety/depression via the activation of the microbiota-gut-brain (MGB) axis [6].

Gut microbiota in humans and animals consist of $>1,000$ bacterial species [7]. Gut bacterial composition fluctuates by endogenous and exogenous factors such as diet, drugs, stressors, and hormones [7,8]. Exposure to IS causes gut dysbiosis, including increased Proteobacteria population, and psychiatric disorders in mice [7, 8]. However, some bifidobacteria and lactobacilli alleviated psychiatric disorders. Lactobacillus reuteri, Lactobacillus plantarum, and Bifidobacterium adolescentis IM38, and Bifidobacterium adolescentis NK98 alleviate anxiety in animals [9-12]. Nevertheless, the effects of gut bacteria on anxiety/ depression still remain unclear.

Therefore, in the present study, we selected BDNF expression-inducing NK41 and NK46 in the gut bacteria collection isolated from human feces using corticosteronestimulated SH-SY5Y cells and examined their effects on ISinduced anxiety/depression in mice.

NK41 and NK46 were cultured in general media for probiotics such as MRS broth (BD, USA), collected by centrifugation $(5,000 \mathrm{~g}, 20 \mathrm{~min})$, and suspended in phosphatebuffered saline (for in vitro study) or 1\% dextrose (for in vivo study) according to the method of Jang et al. [10]. SHSY5Y cells were cultured according to the method of Lee, et al. [13].

All animal experiments were approved by the Institutional Animal Care and Use Committee of the 
University (IACC No KUASP(SE)-18-045) and thereafter carried out according to the NIH and University Guide for Laboratory Animal Care and Usage. Male C57BL/6 mice (age, 6 weeks old; weight, 19-21 g) were supplied by Samtako Inc. (Korea). The mice were kept in the conditions of a controlled temperature of $20 \pm 2{ }^{\circ} \mathrm{C}$, humidity of $50 \pm$ $10 \%$, and light-dark cycle of $12 \mathrm{~h}$. The mice were fed standard laboratory chow with tap water ad libitum.

Anxiety/depression was induced by exposure to IS and anxiety-like and depressive behaviors were measured with elevated plus maze (EPM), forced swimming (FS), and tail suspension (TS) tasks according to the method of Jang et al. [10].

Mice were randomly divided into six groups (Cs, Is, IsL, IsB, IsM, and IsF). Each group consisted of eight mice. Briefly, mice except the Cs group were exposed to IS for $12 \mathrm{~h}$ once a day for 2 days in a cylindrical instrument $(2.5 \times$ $7.5 \mathrm{~cm})$ with a hole $(0.25 \mathrm{~cm}$ in diameter $)$ on the center of the top surface according to the method of Jang et al. [9]. The Cs group was not treated with IS. Test agents (Cs, 1\% dextrose; Is, $1 \%$ dextrose; IsL, $1 \times 10^{9}$ colony-forming units [CFU] of NK41/mouse/day; IsB, $1 \times 10^{9} \mathrm{CFU}$ of NK46/ mouse/day; IsM, $1 \times 10^{9}$ CFU of NK41 and NK46 (1:1, BL) $\mathrm{mix} / \mathrm{mouse} /$ day; IsF, $12 \mathrm{mg}$ of fluoxetine/ $\mathrm{kg} /$ day) were given orally for five days from $24 \mathrm{~h}$ after the final IS treatment.

Immunobloting, enzyme-immunosorbent assay, myeloperoxidase activity, LPS concentration assay, and quantiative real-time polymerase chain reaction for gut bacteria analysis are described in Supplement.

Experimental data are indicated as mean \pm standard deviation (SD). The significant difference was analyzed by one-way analysis of variance followed by a Duncan multiple range test $(p<0.05)$.

We assayed the BDNF expression-inducing activities of gut bacteria isolated from human feces in corticosteronestimulated SH-SY5Y cells. The stimulation of corticosterone suppressed BDNF expression and cAMP response elementbinding protein (CREB) phosphorylation. Of the tested bacteria, NK41 and NK46 potently increased corticosteronesuppressed BDNF expression (Fig. 1). They were identified as Lactobacillus mucosae and Bifidobacterium longum based on the results of Gram staining, a sugar utilization test (API 50 CHL Kit), and 16S rDNA sequencing, respectively.

Next, we orally gavaged NK41, NK46, or their (1:1) mix in mice with IS-induced anxiety/depression and measured their anxiety-like and depressive behaviors in the EPM, TS, and FS tasks (Fig. 2). Exposure to IS significantly decreased

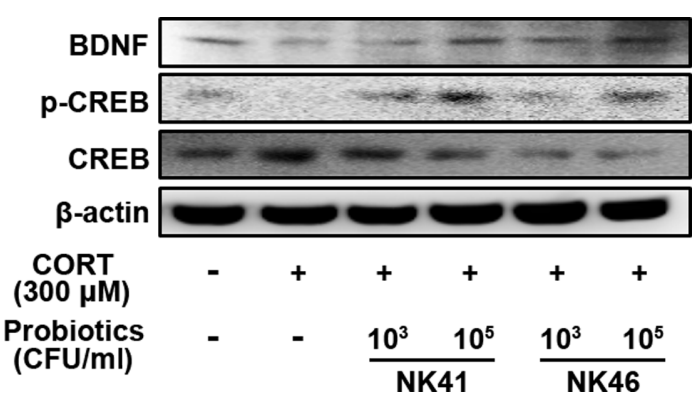

Fig. 1. Effects of Lactobacillus mucosae NK41 and Bifidobacterium longum NK46 on the BDNF expression and CREB phosphorylation in corticosterone-stimulated SH-SY5Y cells.

Cells $\left(1 \times 10^{6}\right)$ were treated with corticosterone $($ CORT, $300 \mu \mathrm{M})$ with or without NK41 or NK46 $\left(1 \times 10^{3}\right.$ or $\left.1 \times 10^{5} \mathrm{CFU} / \mathrm{ml}\right)$ for $24 \mathrm{~h}$. BDNF, CREB, $p$-CREB and $\beta$-actin were measured by immunoblotting.

time spent in the open arms (OT) and open arm entries (OE) in the EPM task compared to those of the control group, respectively. However, treatment with NK41 or NK46 significantly increased IS-suppressed OT and OE. NK41 or NK46 treatment also decreased IS-induced immobility in the FS and TS tasks. Furthermore, treatment with their (1:1) mixture also synergistically or additively suppressed ISinduced, anxiety-like and depressive behaviors in EPM, TS, and FS tasks.

NK41, NK46, and BL mix suppressed IS-induced NF-кB activation in the hippocampus and increased IS-suppressed BDNF expression. Furthermore, they suppressed IS-induced infiltration of $\mathrm{Iba}^{+}$cells (microglia) into the hippocampal CA1 and CA3 regions (Figs. $2 \mathrm{E}$ and S1). Treatment with NK41, NK46, or BL mix decreased IS-induced corticosterone, IL-6, tumor necrosis factor (TNF)- $\alpha$, and LPS levels in the blood.

Next, we examined the anti-colitis effects of NK41, NK46, and BL mix in mice with IS-induced anxiety/depression (Fig. 3). Exposure to IS significantly induced colitis: it induced colon shortening, myeloperoxidase activity, NF- $\kappa \mathrm{B}$ activation, TNF- $\alpha$ and IL- 6 expression, and CD11b ${ }^{+} /$CD45 populations (DCs and macrophages). However, treatment with NK41, NK46, or BL mix significantly suppressed ISinduced colitis markers including colon NF- $\mathrm{\kappa B}$ activation, and $\mathrm{CD} 11 \mathrm{~b}^{+} / \mathrm{CD} 45^{+}$cell population in the colon.

IS exposure increased fecal Bacteroidetes and Proteobacteria populations and reduced the fecal Firmicutes population (Fig. 4). However, their treatment suppressed IS-induced Bacteroidetes and Proteobacteria populations and increased the IS-suppressed Firmicutes population. 
A

(a)

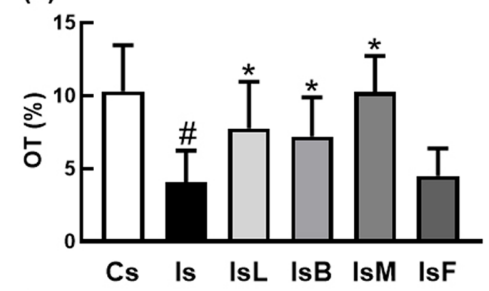

C

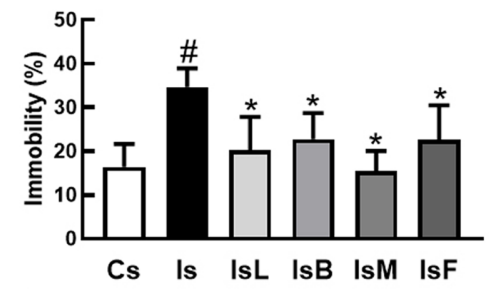

$\mathbf{F}$

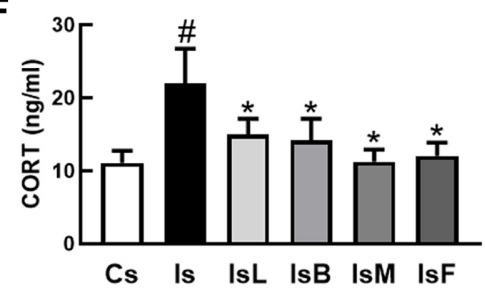

H

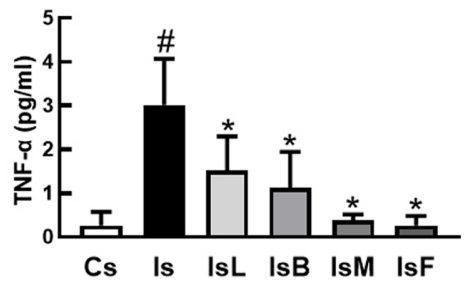

(b)

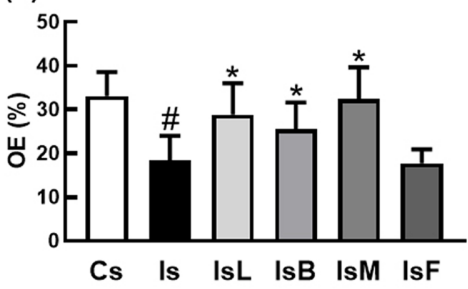

D

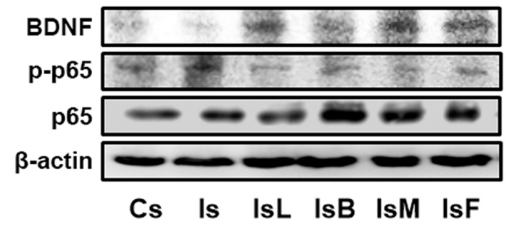

G

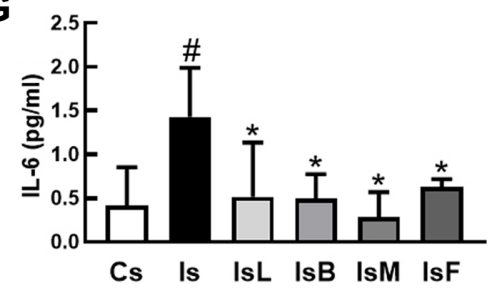

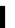

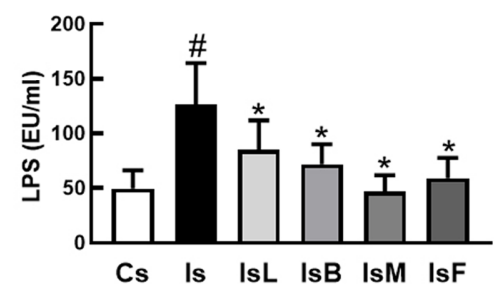

B

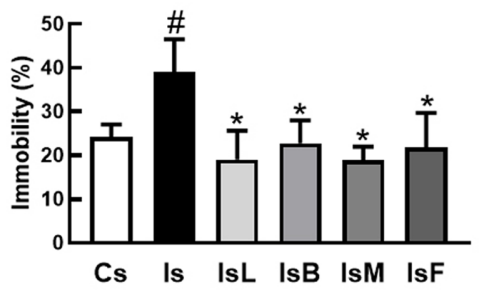

E

CA3 field of hippocampus Magnification $\times 20$
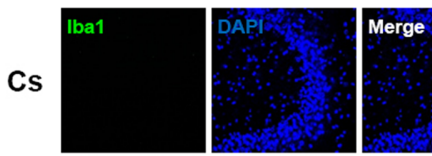

IS
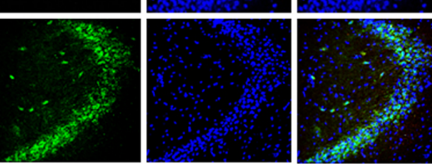

IsL
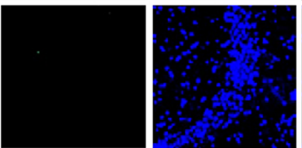

IsB
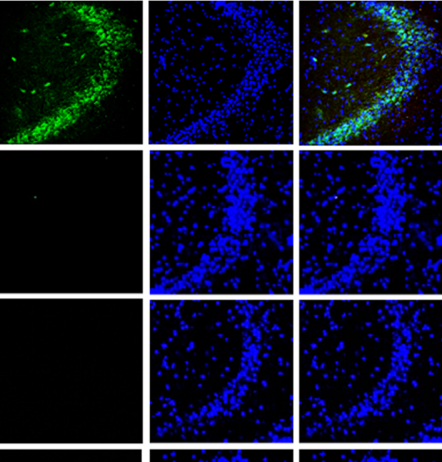

IsM
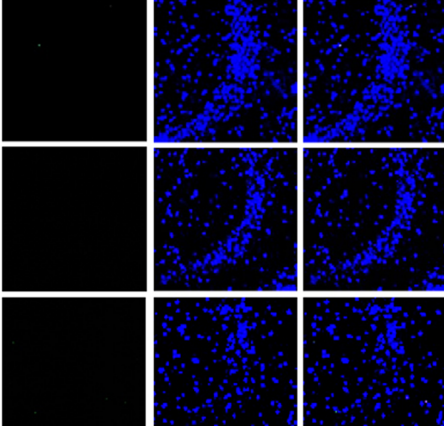

IsF
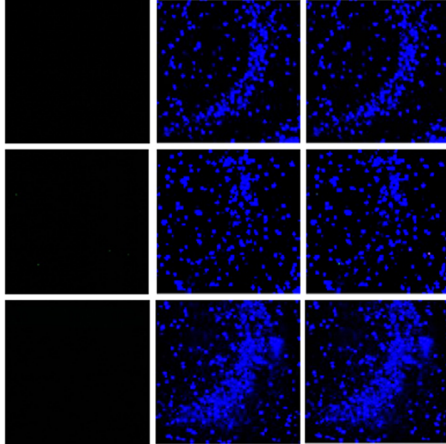

Fig. 2. Effects of Lactobacillus mucosae NK41 and Bifidobacterium longum NK46 on the IS-induced anxiety-like and depressive behaviors and hippocampal inflammation in mice.

Effects on anxiety-like behaviors in the EPM (A: a, OT; b, OE), TST (B), and FST (C) tasks. (D) Effects on NF-kB activation and BDNF expression, in the hippocampus. (E) Effects on the infiltration of Iba1 ${ }^{+}$cells into the CA3 region of hippocampus. Effects on corticoterone (CORT, F), IL-6 (G), TNF- $\alpha(\mathbf{H})$, and LPS levels (I) in the blood. Mice except control group (Cs) were exposed to IS and test agents (Cs, vehicle [1\% dextrose]; Is, vehicle; IsL, $1 \times 10^{9} \mathrm{CFU} /$ mouse/day of NK41; IsB, $1 \times 10^{9} \mathrm{CFU} / \mathrm{mouse} /$ day of NK46; IsM, $1 \times 10^{9} \mathrm{CFU} / \mathrm{mouse} /$ day of the $(1: 1)$ mixture of NK41 and NK46 (BL mix)]; and IsF, $12 \mathrm{mg} / \mathrm{kg} /$ day of fluoxetine) were gavaged orally once a day for 5 days. p65, p-p65, BDNF, and $\beta$-actin were measured by immunoblotting. Blood corticosterone, IL-6, TNF- $\alpha$, and LPS were assayed by ELISA kits. Iba1 ${ }^{+}$cells were observed by a confocal microscope. All data were expressed as mean $\pm \mathrm{SD}(n=8) .{ }^{*} p<0.05$ vs. Cs group. ${ }^{*} p<0.05$ vs. IS-treated control (IS) group.

They also suppressed the IS-induced gut bacterial LPS production.

Gut microbiota are closely associated with the occurrence of psychiatric disorders such as Alzheimer's disease and anxiety/depression [7, 14, 15]. Germ-free or antibioticstreated mice exhibit exaggerated anxiety-like behaviors more than conventional mice [16]. These behaviors are alleviated by fecal transplantation from conventional mice. Exposure to stressors such as IS and 2,4,6-trinitrobenzenesulfonic acid causes gut inflammation and membrane permeability $[6,17]$.

In the present study, exposure to IS induced anxiety/ depression, NF- $\kappa \mathrm{B}$ activation in the hippocampus and colon, and gut Proteobacteria population and bacterial LPS 
A

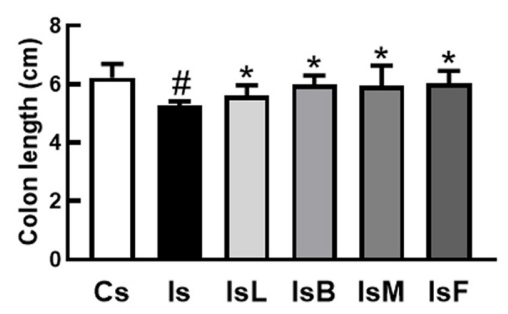

D

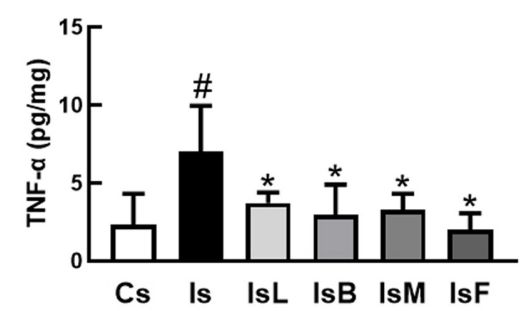

F

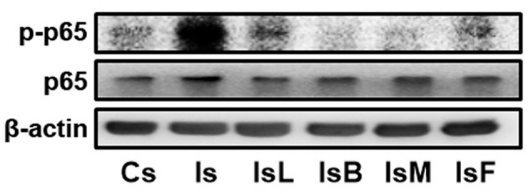

B

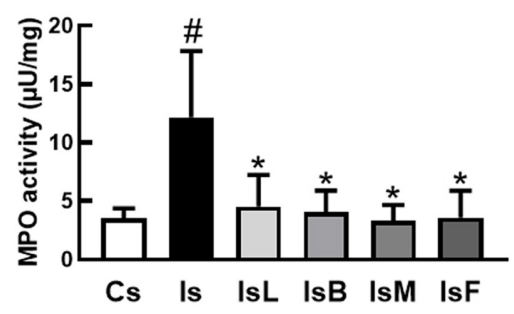

E
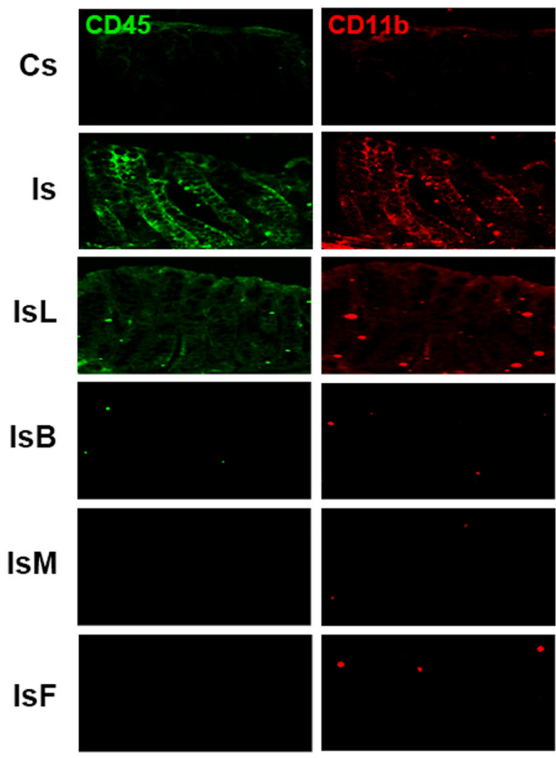

C

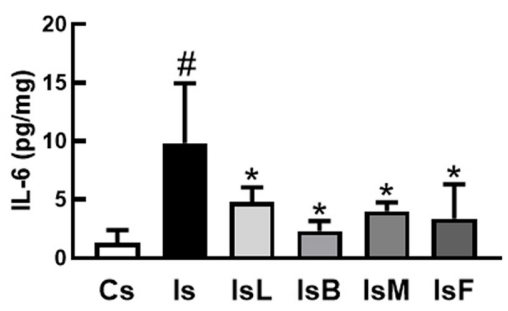

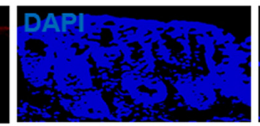

Merge
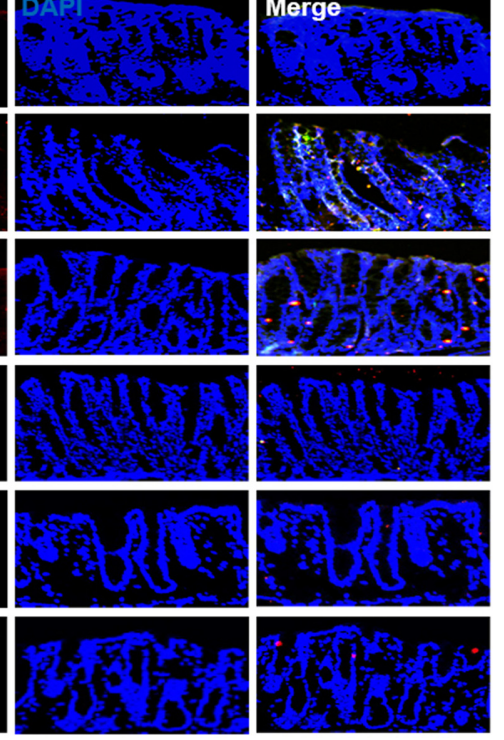

Fig. 3. Effects of Lactobacillus mucosae NK41 and Bifidobacterium longum NK46 on the colitis in mice with IS-induced anxiety/ depression.

Effects on colon length (A), myeloperoxidase (MPO) activity, IL-6 (C) and TNF- $\alpha$ expression (D). Effects on CD11b ${ }^{+}$CD45 $5^{+}$cell population (E) and NF- $\kappa$ B activation (F). Mice except control group (Cs) were exposed to IS and test agents (Cs, vehicle [1\% dextrose]; Is, vehicle; IsL, $1 \times 10^{9} \mathrm{CFU} /$ mouse/day of NK41; IsB, $1 \times 10^{9} \mathrm{CFU} /$ mouse/day of NK46; IsM, $1 \times 10^{9} \mathrm{CFU} / \mathrm{mouse} /$ day of the (1:1) mixture of NK41 and NK46 (BL mix)]; and IsF, $12 \mathrm{mg} / \mathrm{kg} /$ day of fluoxetine) were gavaged orally once a day for 5 days. p65, p-p65, and $\beta$-actin were measured by immunoblotting. IL-6 and TNF- $\alpha$ expression were assayed by ELISA kits. CD11b ${ }^{+} / \mathrm{CD} 45^{+}$cells were observed by a confocal microscope. All data were expressed as mean \pm $\mathrm{SD}(n=8) .{ }^{\#} p<0.05$ vs. Cs group. ${ }^{*} p<0.05$ vs. IS-treated control (IS) group.

production, as previously reported [6,9]. Oral administration of NK41, NK46, or BL mix significantly alleviated ISinduced anxiety/depression, NF- $\kappa$ B activation, and gut dysbiosis. In particular, they suppressed IS-induced gut bacterial LPS production and endotoxemia. LPS causes systemic inflammation and psychiatric disorders such as memory impairment and depression and suppresses BDNF and tight junction protein expression in vitro and in vivo $[6,11]$. These findings suggest that NK41 and NK46 can alleviate anxiety/depression by inhibiting gut inflammation through the inhibition of gut bacterial LPS production.
Lactobacillus johnsonii and Lactobacillus plantarum alleviate anxiety in mice and zebrafish, respectively [6, 11]. Bifidobacterium longum NCC3001 mitigated dextran sodium sulfate-induced anxiety in rats [19]. Bifidobacterium infantis suppresses depression in the brainstems of rats [18]. Antiinflammatory Lactobacillus reuteri NK33 and Bifidobacterium adolescentis NK98 attenuated IS-induced depression and colitis by inhibiting NF- $\kappa$ B activation.

These findings suggest that probiotics NK41 and NK46 may alleviate anxiety/depression and colitis by suppressing gut dysbiosis. 
A

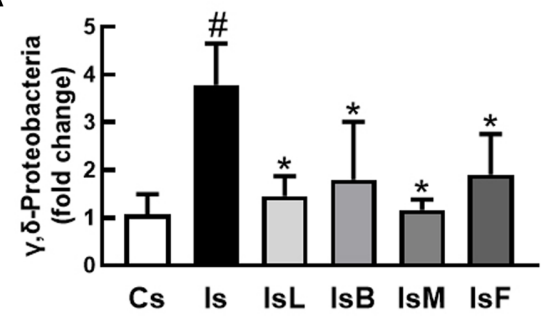

C

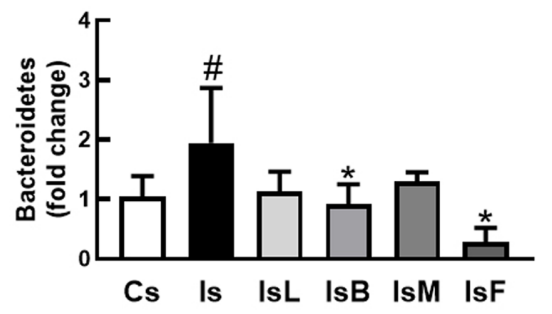

B

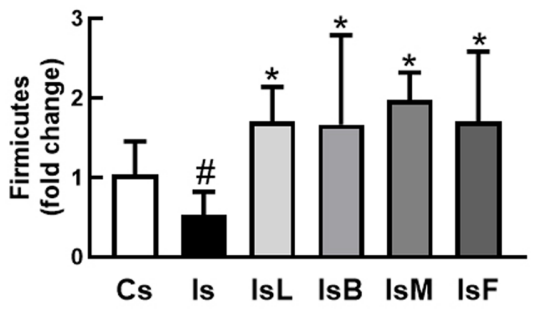

D

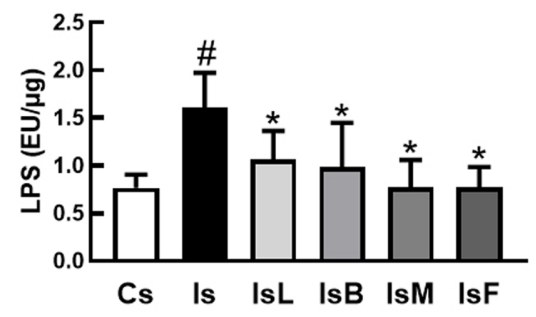

Fig. 4. Effects of Lactobacillus mucosae NK41 and Bifidobacterium longum NK46 on the gut bacteria and LPS production in mice with IS-induced anxiety/depression.

Gut bacteria Proteobacteria (A), Firmicutes (B), and Bacteroidetes populations (C) were assayed by qPCR. Bacterial LPS was measured by ELISA. (D) Effects on the fecal LPS level. Mice except control group (Cs) were exposed to IS and test agents (Cs, vehicle [1\% dextrose]; Is, vehicle; IsL, $1 \times$ $10^{9} \mathrm{CFU} /$ mouse/day of NK41; IsB, $1 \times 10^{9} \mathrm{CFU} /$ mouse/day of NK46; IsM, $1 \times 10^{9} \mathrm{CFU} /$ mouse/day of the (1:1) mixture of NK41 and NK46 (BL $\mathrm{mix})$ ]; and IsF, $12 \mathrm{mg} / \mathrm{kg} /$ day of fluoxetine) were gavaged orally once a day for 5 days. All data were expressed as mean $\pm \mathrm{SD}(n=8){ }^{*} p<0.05 \mathrm{vs}$. Cs group. ${ }^{*} p<0.05$ vs. IS-treated control (IS) group.

\section{Acknowledgement}

We thank Hyo-Min Jang for the technical assistance. This work was supported by the Korea Institute of Planning and Evaluation for Technology in Food, Agriculture, Forestry (IPET) through the High Value-added Food Technology Development Program, funded by the Ministry of Agriculture, Food and Rural Affairs (MAFRA) (318027-4).

\section{Conflicts of Interest}

The authors have no financial conflicts of interest to declare.

\section{References}

1. Baxter AJ, Scott KM, Ferrari AJ, Norman RE, Vos T, Whiteford HA. 2014. Challenging the myth of an "epidemic" of common mental disorders: trends in the global prevalence of anxiety and depression between 1990 and 2010. Depress. Anxiety 31: 506-516.

2. Hart PC, Bergner CL, Smolinsky AN, Dufour BD, Egan RJ, Laporte JL, et al. 2016. Experimental models of anxiety for drug discovery and brain research. Methods Mol. Biol. 1438: 271-291.
3. Sutanto D, Kloet ER. 1994. The use of various animal models in the study of stress and stress-related phenomena. Lab. Anim. 28: 293-306.

4. Sekiyama A, Ueda H, Kashiwamura S, Nishida K, Yamaguchi S, Sasaki H, et al. 2006. A role of the adrenal gland in stress-induced up-regulation of cytokines in plasma. J. Neuroimmunol. 171: 38-44.

5. Caviedes A, Lafourcade C, Soto C, Wyneken U. 2017. $\mathrm{BDNF} / \mathrm{NF}-\kappa \mathrm{B}$ signaling in the neurobiology of depression. Curr. Pharm. Des. 23: 3154-3163.

6. Jang HM, Lee KE, Lee HJ, Kim DH. 2018. Immobilization stress-induced Escherichia coli causes anxiety by inducing NF-кB activation through gut microbiota disturbance. Sci. Rep. 8: 13897.

7. Hold GL, Hansen R. 2019. Impact of the gastrointestinal microbiome in health and disease: co-evolution with the host immune system. Curr. Top. Microbiol. Immunol. 421: 303318.

8. Molina-Torres G, Rodriguez-Arrastia M, Roman P, SanchezLabraca N, Cardona D. 2019. Stress and the gut microbiotabrain axis. Behav. Pharmacol. 30: 187-200.

9. Jang HM, Lee KE, Kim DH. 2019. The preventive and curative effects of Lactobacillus reuteri NK33 and Bifidobacterium adolescentis NK98 on immobilization stress-induced anxiety/ depression and colitis in mice. Nutrients 11: pii: E819. 
10. Jang HM, Jang SE, Han MJ, Kim DH. 2018. Anxiolytic-like effect of Bifidobacterium adolescentis IM38 in mice with or without immobilisation stress. Benef. Microbes. 9: 123-132.

11. Davis DJ, Doerr HM, Grzelak AK, Busi SB, Jasarevic E, Ericsson AC, et al. 2016. Lactobacillus plantarum attenuates anxiety-related behavior and protects against stress-induced dysbiosis in adult zebrafish. Sci. Rep. 6: 33726.

12. Liu X, Cao S, Zhang X. 2015. Modulation of gut microbiotabrain axis by probiotics, prebiotics, and diet. J. Agric. Food Chem. 63: 7885-7895.

13. Lee HJ, Lim SM, Ko DB, Jeong JJ, Hwang YH, Kim DH. 2017. Soyasapogenol B and genistein attenuate lipopolysaccharideinduced memory impairment in mice by the modulation of NF-kB-mediated BDNF expression. J. Agric. Food Chem. 65: 6877-6885.

14. Jang HM, Lee HJ, Jang SE, Han MJ, Kim DH. 2018. Evidence for interplay among antibacterial-induced gut microbiota disturbance, neuro-inflammation, and anxiety in mice. Mucosal Immunol. 11: 1386-1397.
15. Mackos AR, Varaljay VA, Maltz R, Gur TL, Bailey MT. 2016. Role of the intestinal microbiota in host responses to stressor exposure. Int. Rev. Neurobiol. 131: 1-19.

16. Sudo N, Chida Y, Aiba Y, Sonoda J, Oyama N, Yu XN, et al. 2004. Postnatal microbial colonization programs the hypothalamic-pituitary-adrenal system for stress response in mice. J. Physiol. 558: 263-275.

17. Jang SE, Lim SM, Jeong JJ, Jang HM, Lee HJ, Han MJ, et al. 2018. Gastrointestinal inflammation by gut microbiota disturbance induces memory impairment in mice. Mucosal Immunol. 11: 369-379.

18. Bercik P, Park AJ, Sinclair D, Khoshdel A, Lu J, Huang X, et al. 2011. The anxiolytic effect of Bifidobacterium longum NCC3001 involves vagal pathways for gut-brain communication. Neurogastroenterol. Motil. 23: 1132-1139.

19. Desbonnet L, Garrett L, Clarke G, Kiely B, Cryan JF, Dinan TG. 2010. Effects of the probiotic Bifidobacterium infantis in the maternal separation model of depression. Neuroscience 170: $1179-1188$. 